\title{
Convex-Meniscus-Assisted Self-Assembly at the Air/Water Interface to Prepare a Wafer-Scale Colloidal Monolayer Without Overlap
}

Xin Li ${ }^{1,2,3}$, Yijun Zhang ${ }^{1,2}$, Mingxiao Li ${ }^{1}$, Yang Zhao ${ }^{1}$, Lingqian Zhang ${ }^{1,}$, and Chengjun Huang ${ }^{1,2,3, *}$

1 R\&D Center of Healthcare Electronics, Institute of Microelectronics, Chinese Academy of Sciences, Beijing 100029, China;

2 University of Chinese Academy of Sciences, Beijing 100049, China;

3 School of Future Technology, University of Chinese Academy of Sciences, Beijing, 100049, China;

* Correspondence: zhanglingqian@ime.ac.cn and huangchengjun@ime.ac.cn 


\section{Contents}

Figure S1. The contact angle of the plasma-treated glass slide.

Figure S2. The photograph of the AWI when colloidal suspension was added.

Figure S3. The image of the concentric circular structure caused by harsh compression to PS nanospheres.

Figure S4. The NTA result and the SEM image of the polystyrene (PS) nanospheres.

Figure S5. The SEM images of the colloidal monolayers self-assembled with the convex-meniscus-assisted protocol and transferred to vertical substrates.

Table S1. The raw data of the number of overlapping nanospheres in different samples.

Video S1. The stable convex meniscus adjacent to the three-phase boundary.

Video S2. The self-assembly process near the three-phase boundary. 


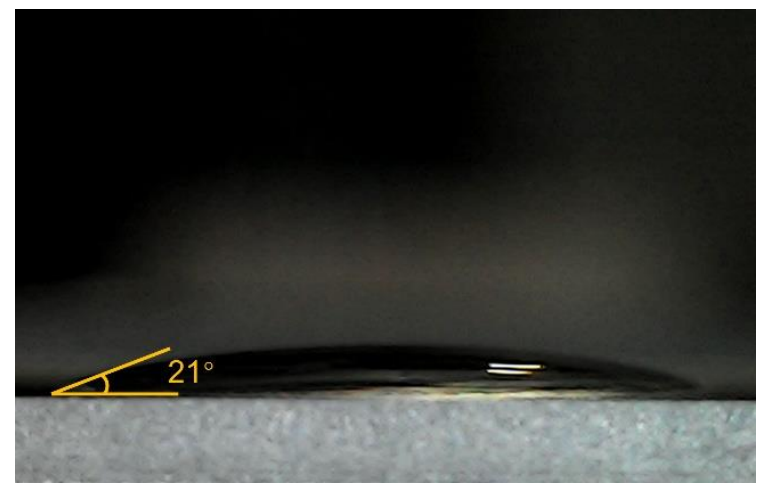

Figure S1. Optical image of a water drop $(20 \mu \mathrm{L})$ on the plasma-treated glass slide showing a contact angle of $21^{\circ}$.
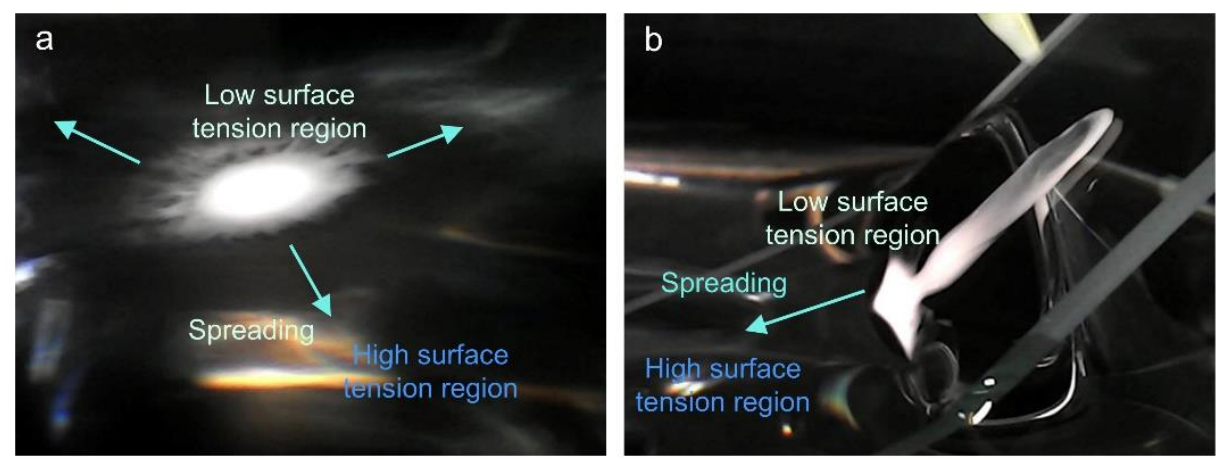

Figure S2. The photograph when the colloidal suspension was added to the AWI. Whether the colloidal suspension was directly dropped to the AWI through a pipette (a), or indirectly released with the assistance of a glass slide (b), the PS nanospheres rapidly spread outward from the regions with low surface tension due to Marangoni effect. 


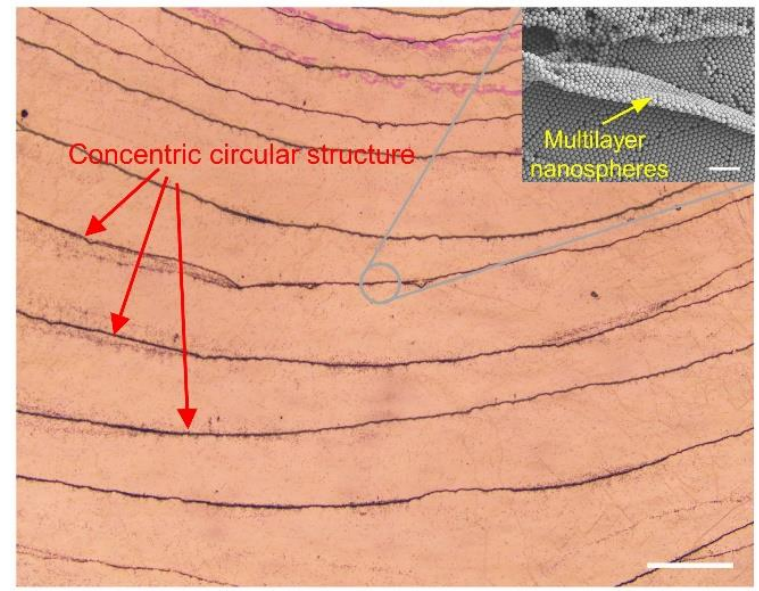

Figure S3. Microphotograph of the concentric circular structure caused by harsh compression of the PS nanospheres. Scale bar: $400 \mu \mathrm{m}$. Inset is the corresponding SEM image, from which the multilayer structure composed of PS nanospheres could be clearly observed. Scale bar: $3 \mu \mathrm{m}$. 

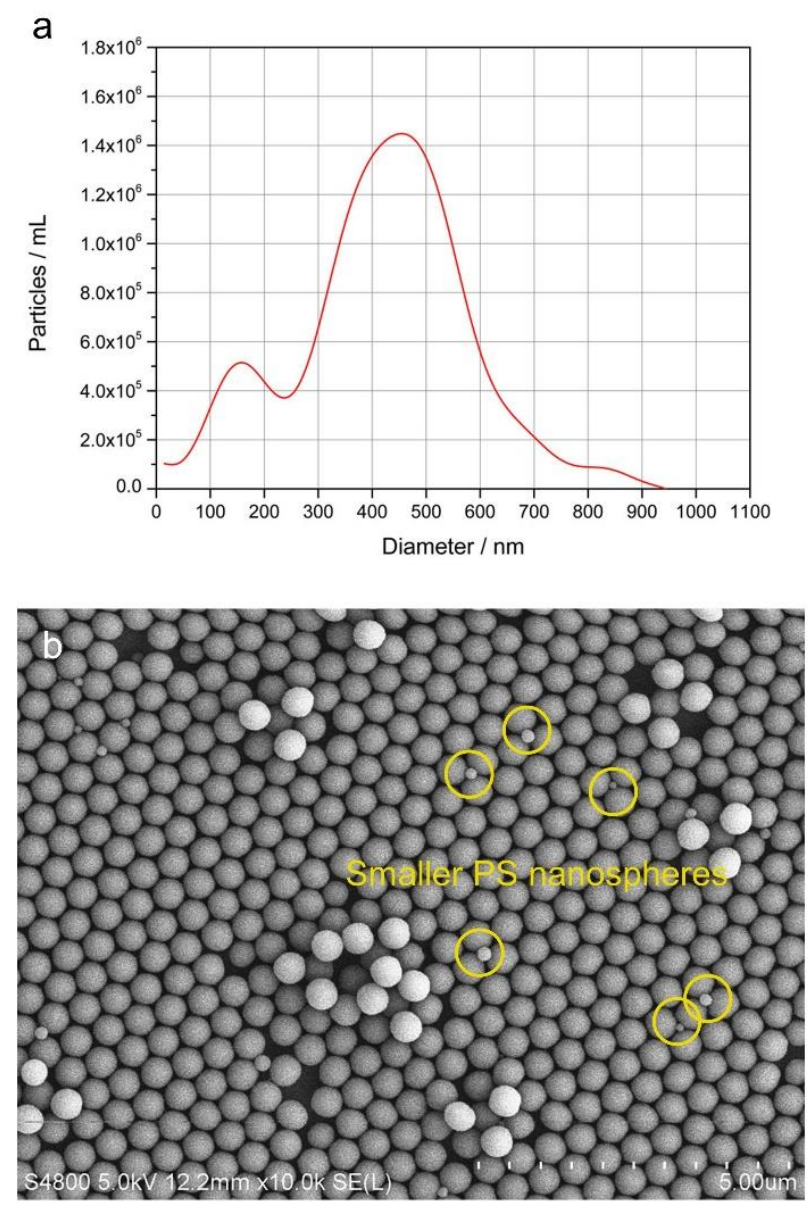

Figure S4. (a). The NTA result of the centration and diameter of the submerged particles. A peak was detected at $150 \mathrm{~nm}$ because of the polydispersity of the PS nanospheres. (b) The SEM of the PS nanospheres with a diameter of around $150 \mathrm{~nm}$. 

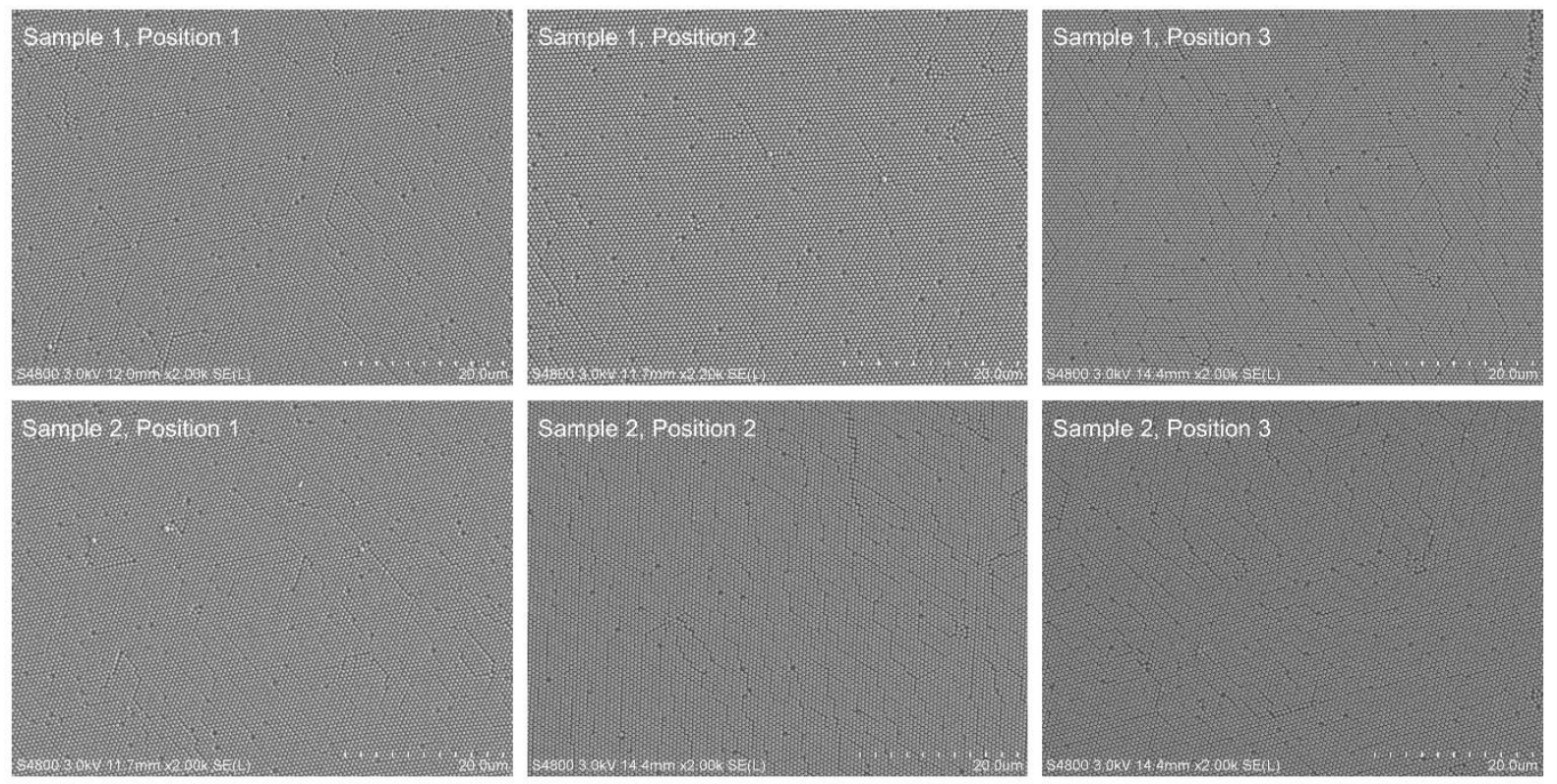

Figure S5. The SEM images of two colloidal monolayers (row 1 and row 2 respectively) selfassembled with the convex-meniscus-assisted protocol and transferred to vertical substrates. No random overlapping nanospheres were observed on the monolayer, showing excellent consistency and repeatability in terms of control on the monolayer morphology.

Table S1. The number of overlapping nanospheres in different samples.

\begin{tabular}{|c|c|c|c|c|c|c|c|c|c|c|c|c|c|c|}
\hline \multirow{2}{*}{$\begin{array}{c}\text { Substrate } \\
\text { inclination } \\
\text { angle }\end{array}$} & \multirow{2}{*}{ Method } & \multicolumn{9}{|c|}{$\begin{array}{c}\text { Position } \\
(64 \mu \mathrm{m} \times 48 \mu \mathrm{m})\end{array}$} & \multirow{2}{*}{$\begin{array}{c}\text { Average } \\
\text { number of } \\
\text { overlapping } \\
\text { nanospheres } \\
\text { in scope }\end{array}$} & \multirow{2}{*}{$\begin{array}{l}\text { Standard } \\
\text { deviation }\end{array}$} & \multirow{2}{*}{$\begin{array}{c}\text { Normalized } \\
\text { average } \\
\text { number } \\
(100 \mu \mathrm{m} \times \\
100 \mu \mathrm{m})\end{array}$} & \multirow{2}{*}{$\begin{array}{c}\text { Normalized } \\
\text { standard } \\
\text { deviation }\end{array}$} \\
\hline & & 1 & 2 & 3 & 4 & 5 & 6 & 7 & 8 & 9 & & & & \\
\hline \multirow{2}{*}{$30^{\circ}$} & Pipette & 114 & 75 & 81 & 108 & 90 & 150 & 300 & 188 & 374 & 164.44 & 105.65 & 535.30 & 343.90 \\
\hline & $\begin{array}{l}\text { Convex } \\
\text { meniscus }\end{array}$ & 0 & 3 & 6 & 9 & 8 & 7 & 6 & 6 & 3 & 5.33 & 2.83 & 17.36 & 9.21 \\
\hline \multirow{2}{*}{$60^{\circ}$} & Pipette & 45 & 60 & 84 & 84 & 57 & 156 & 75 & 84 & 129 & 86.00 & 35.50 & 279.95 & 115.55 \\
\hline & $\begin{array}{l}\text { Convex } \\
\text { meniscus }\end{array}$ & 6 & 0 & 0 & 3 & 0 & 0 & 2 & 5 & 0 & 1.78 & 2.39 & 5.79 & 7.77 \\
\hline \multirow{2}{*}{$90^{\circ}$} & Pipette & 75 & 3 & 6 & 0 & 6 & 3 & 3 & 30 & 53 & 19.89 & 27.09 & 64.74 & 88.20 \\
\hline & $\begin{array}{l}\text { Convex } \\
\text { meniscus }\end{array}$ & 1 & 0 & 0 & 1 & 0 & 0 & 0 & 0 & 0 & 0.22 & 0.44 & 0.72 & 1.44 \\
\hline
\end{tabular}

